\section{OPEN ACCESS}

Edited by:

Gregoire P. Millet,

University of Lausanne, Switzerland

Reviewed by:

Samuele Maria Marcora, University of Kent, United Kingdom Andrew Renfree

University of Worcester United Kingdom

*Correspondence: Louise M. Burke louise.burke@ausport.gov.au

Specialty section

This article was submitted to

Exercise Physiology,

a section of the journal

Frontiers in Physiology

Received: 22 May 2017 Accepted: 29 September 2017 Published: 23 October 2017

Citation:

Leckey JJ, Ross ML, Quod M, Hawley JA and Burke LM (2017) Ketone Diester Ingestion Impairs

Time-Trial Performance in Professional

Cyclists. Front. Physiol. 8:806. doi: 10.3389/fphys.2017.00806

\title{
Ketone Diester Ingestion Impairs Time-Trial Performance in Professional Cyclists
}

\begin{abstract}
Jill J. Leckey ${ }^{1}$, Megan L. Ross ${ }^{1,2}$, Marc Quod ${ }^{3}$, John A. Hawley ${ }^{1,4}$ and Louise M. Burke ${ }^{1,2 *}$
${ }^{1}$ Centre for Exercise and Nutrition, Mary MacKillop Institute for Health Research, Australian Catholic University, Melbourne, VIC, Australia, ${ }^{2}$ Sports Nutrition, Australian Institute of Sport, Canberra, ACT, Australia, ${ }^{3}$ ORICA-BikeExchange WorldTour Team), UCl, Adelaide, SA, Australia, ${ }^{4}$ Research Institute for Sport and Exercise Sciences, Liverpool John Moores University, Liverpool, United Kingdom
\end{abstract}

We investigated the effect of pre- "race" ingestion of a 1,3-butanediol acetoacetate diester on blood ketone concentration, substrate metabolism and performance of a cycling time trial (TT) in professional cyclists. In a randomized cross-over design, 10 elite male cyclists completed a $\sim 31 \mathrm{~km}$ laboratory-based $\Pi \mathrm{T}$ on a cycling ergometer programmed to simulate the 2017 World Road Cycling Championships course. Cyclists consumed a standardized meal [2 g/kg body mass (BM) carbohydrate $(\mathrm{CHO})$ ] the evening prior to a trial day and a $\mathrm{CHO}$ breakfast ( $2 \mathrm{~g} / \mathrm{kg} \mathrm{BM} \mathrm{CHO}$ ) with $200 \mathrm{mg}$ caffeine on the morning of a trial day. Cyclists were randomized to consume either the ketone diester $(2 \times 250 \mathrm{mg} / \mathrm{kg})$ or a placebo drink, followed immediately by $200 \mathrm{~mL}$ diet cola, given $\sim 30$ min before and immediately prior to commencing a 20 min incremental warm-up. Blood samples were collected prior to and during the warm-up, pre- and post- $\Pi$ and at regular intervals after the $\Pi$. Urine samples were collected pre- and post- warm-up, immediately post $\Pi$ and 60 min post $\Pi$. Pre-exercise ingestion of the diester resulted in a $2 \pm 1 \%$ impairment in $T$ performance that was associated with gut discomfort and higher perception of effort. Serum $\beta$-hydroxybutyrate, serum acetoacetate, and urine ketone concentrations increased from rest following ketone ingestion and were higher than placebo throughout the trial. Ketone ingestion induces hyperketonemia in elite professional cyclists when in a carbohydrate fed state, and impairs performance of a cycling $\Pi$ lasting $\sim 50 \mathrm{~min}$.

\section{Keywords: ketone ester, cycling, time-trial, performance, ketones}

\section{INTRODUCTION}

Substrate utilization during exercise is influenced by several factors including the relative intensity and duration of exercise, an individual's training status and the effect of the preceding diet on both the substrate pool and the prevailing hormonal milieu (Hawley et al., 2015). As exercise intensity increases, there is a greater reliance on carbohydrate (CHO) based fuels (i.e., muscle and liver glycogen, blood glucose, lactate) and a reduction in the utilization of fat substrates (Brooks and Mercier, 1994). Ketone bodies provide another potential source of readily oxidized fuel for skeletal muscle, but are predominately associated with conditions of metabolic stress such as starvation, where they are needed to preserve essential function of peripheral tissues including the brain and heart (Robinson and Williamson, 1980; Veech, 2004). However, there has been recent interest 
in the notion that increasing blood ketone concentrations could contribute to an enhancement of exercise performance by providing a readily available, alternative oxidative substrate for working muscle, sparing the limited stores of muscle glycogen (Pinckaers et al., 2017). As such, models of hyperketonemia from endogenous origin such as chronic exposure to a ketogenic diet (Burke et al., 2017) as well as introduction of exogenous sources of ketone bodies from ketone ester supplements (Cox et al., 2016) have been investigated. Nevertheless, there is some concern that the presence of high circulating concentrations of ketone bodies could inhibit the flux of other muscle substrates, either by impairing (rather than "sparing") muscle $\mathrm{CHO}$ oxidation and/or inhibiting adipose tissue lipolysis (Evans et al., 2017). As such, the situations in which an available ketone supply may benefit exercise capacity or performance may be determined by the duration and/or intensity of exercise and the need for combinations of muscle substrate to meet the metabolic demands.

Ketone bodies, namely D- $\beta$-hydroxybutyrate $(\beta \mathrm{HB})$, acetone and acetoacetate (AcAc), are produced in the liver mitochondria from acetyl-CoA in response to an increased mobilization of free fatty acids (FFA) from adipose tissue in situations of reduced CHO availability (Robinson and Williamson, 1980). As summarized in recent reviews (Egan and D'Agostino, 2016; Pinckaers et al., 2017), under conditions of high CHO availability, circulating concentrations of ketone bodies are low, but are slightly elevated $(0.1-0.5 \mathrm{mmol} / \mathrm{L})$ by an overnight fast and further raised by exercising in a fasted state $(0.5-1.0 \mathrm{mmol} / \mathrm{L})$. Prolonged fasting/starvation (5 days) causes a maximal increase in rates of ketone body production (1-2 mmol/min or $140-280$ $\mathrm{g} /$ day), leading to increased plasma concentrations that plateau under normal physiological conditions at $\sim 7-10 \mathrm{mmol} / \mathrm{L}$. Meanwhile, chronic exposure to a ketogenic diet [low $\mathrm{CHO}(<50$ $\mathrm{g} / \mathrm{d})$, low-moderate protein $(\sim 15 \%$ of energy), high fat $(75-$ $80 \%$ of energy)] raises plasma ketone bodies to $1-2 \mathrm{mmol} / \mathrm{L}$ after several days, with concentrations reaching the apparent plateau achieved by prolonged fasting, according to the level of $\mathrm{CHO}$ restriction and duration of "keto-adaptation" (Pinckaers et al., 2017). Exogenous forms of ketone bodies include ketone salts, and more recently, ketone esters. Ingestion of the former appears to be less effective in increasing circulating ketone body concentrations and carries a significant salt load (Balasse and Ooms, 1968). Recently, a newly produced ketone monoester, R-3hydroxybutyl R-3-hydroxybutyrate (Clarke et al., 2012), increases in plasma ketone concentrations $(3-6 \mathrm{mmol} / \mathrm{L})$ within the hours following its ingestion (400-600 $\mathrm{mg} / \mathrm{kg} \mathrm{BM}$ ), although this may be altered by concomitant intake of food (Evans et al., 2017; Pinckaers et al., 2017).

To investigate the potential benefits to metabolism and sports performance, Cox et al. (2016) studied the effects of ingesting either $\mathrm{CHO}$ or $\mathrm{CHO}$ plus ketone ester $\left(573 \mathrm{mg} \cdot \mathrm{kg}^{-1} \mathrm{BM}\right)$ on performance in trained cyclists. Their ingestion protocol induced a higher blood $\mathrm{D}-\beta \mathrm{HB}$ concentration during submaximal cycling (ranging between $\sim 1.5$ and $3 \mathrm{mmol} / \mathrm{L}$ ) and lead to a subsequent improvement in time-trial (TT) performance by $\sim 2 \%$ following ketone ester and $\mathrm{CHO}$ ingestion compared to the ingestion of only $\mathrm{CHO}$. However, aspects of the study design are inconsistent with conditions of "real world" cycling competition. Accordingly, we examined the effect of pre-"race" ingestion of a ketone diester on blood ketone body concentrations, substrate metabolism and performance under conditions of elite professional cycling; ingestion of a pre-race $\mathrm{CHO}$-rich meal, inclusion of a warm-up, involvement of world-class cyclists and simulation of a real-life course. We hypothesized that this protocol would result in acute nutritional ketosis but that no performance improvement would be observed due to the high intensity nature of a real-life TT event which is dependent on the high rates of energy production from the oxidation of $\mathrm{CHO}$-based fuels.

\section{MATERIALS AND METHODS}

\section{Ethical Approval}

This study conformed to the standards set by the Declaration of Helsinki and was approved (\#20161005) by the Ethics Committee of the Australian Institute of Sport (AIS). After comprehensive details of the study protocol were explained to the participants verbally and in writing, all participants provided written informed consent.

\section{Overview of Study Design}

The study was a randomized crossover, double-blind, placebocontrolled design using elite (professional) cyclists attending a pre-season camp at the AIS, Canberra. On two separate occasions, three days apart, participants completed a 20-min standardized warm-up and rested for 5 min prior to completing a $31 \mathrm{~km}$ TT performed on a cycling ergometer (Figure 1). Participants were randomized to consume a 1,3-butanediol acetoacetate diester (described subsequently; KET; two doses of $250 \mathrm{mg} / \mathrm{kg} \mathrm{BM}$ ) or a viscosity and color-matched (PLAC) drink, given $\sim 30$ min before and immediately prior to commencing the warm up. It was not possible to completely replicate the taste of the KET drink, but a comparably novel and bitter-tasting PLAC was prepared from a mixture of flavor essences (rum, almond, and bitters Angostura). Pilot testing revealed that the intake of a small volume of diet cola immediately after the KET and PLAC was able to quickly mask the taste and texture of the previous drink. In any case, none of the participants had previously ingested a ketone ester supplement and were therefore unable to recognize its characteristics. In recognition of the World Anti-Doping Code under which these cyclists compete, it was ascertained that ketone supplements are not considered a prohibited substance by the World Anti-Doping Agency.

\section{Participants}

Eleven internationally competitive male cyclists [age, $25 \pm 7$ $(S D)$ y; body mass (BM), $73.7 \pm 7.6 \mathrm{~kg}$; $\dot{V}_{2}$ peak, $71.4 \pm 5.6$ $\mathrm{mL} / \mathrm{kg} / \mathrm{min}, 5.3 \pm 0.3 \mathrm{~L} / \mathrm{min}$; Maximal Aerobic Power (MAP), $494 \pm 20 \mathrm{~W}]$ from the ORICA-BikeExchange UCI World Tour (Road Cycling) team participated in this study. Participants included world class elite $(n=8$; e.g., 2016 Paris-Roubaix winner, stage medalists from Tour de France, Tour Down Under, Giro d'Italia, Vuelta a España and Australian National championship Time Trial medalists) and highly trained under 23 riders contracted to the team $(n=3)$. 

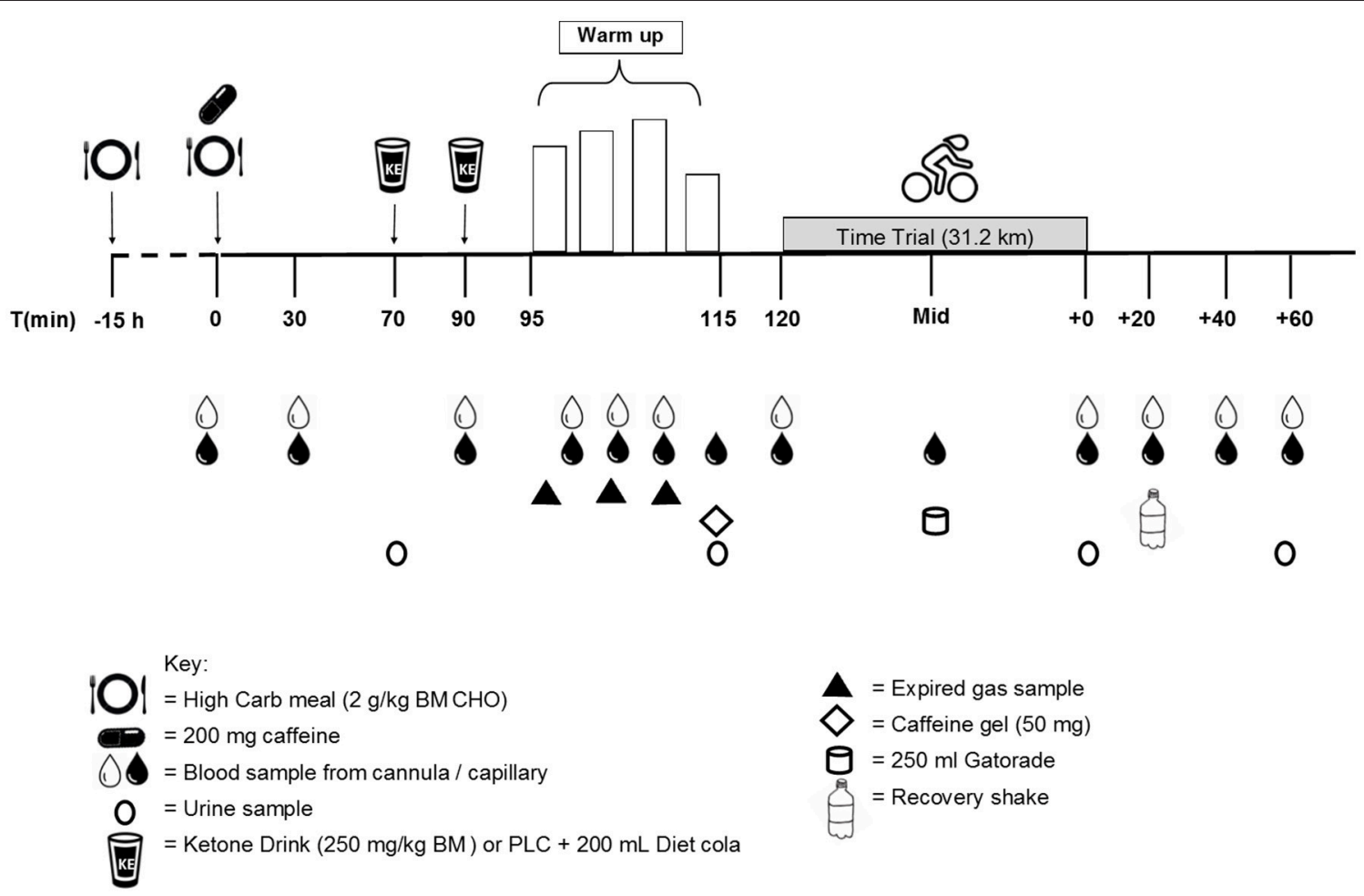

FIGURE 1 | Overview of study design.

\section{Preliminary Testing and Familiarization}

Before commencing the experimental phase, participants visited the laboratory to complete an incremental exercise test and a familiarization with the cycling ergometer (Velotron, Racermate Inc., Seattle, WA, USA) and the experimental exercise protocol (simulated 2017 World Championships time trial course, Bergen Norway).

\section{Incremental Cycling Test}

Participants completed a 5-min warm-up at $150 \mathrm{~W}$ on the cycle ergometer (Lode Excalibur Sport, Groningen, The Netherlands), thereafter the test protocol started at $180 \mathrm{~W}$ and increased $30 \mathrm{~W}$ every $60 \mathrm{~s}$ until volitional exhaustion. MAP was determined as the power output $(\mathrm{PO})$ of the highest stage completed. If the participant finished partway through a $60 \mathrm{~s}$ stage, MAP was calculated in a pro-rata manner. During the maximal test, expired gases were collected into a calibrated and customized Douglas bag gas analysis system, as previously described (Russell et al., 2002). Peak aerobic capacity ( $\mathrm{V}_{2}$ peak) was calculated as the highest oxygen consumption recorded over a 30-s period. $\dot{\mathrm{V}} \mathrm{O}_{2}$ peak and MAP were used to calculate the PO for the individualized warm up on subsequent trial days, described below.

\section{Dietary Control}

$\mathrm{CHO}$ and caffeine intake were standardized the evening before and morning of a trial day and participants were also instructed to abstain from alcohol during the $24 \mathrm{~h}$ period prior to a trial day. Participants consumed an evening meal, snack and breakfast prepared by the team chef, providing a $\mathrm{CHO}$ content of 2,1 , and $2 \mathrm{~g} / \mathrm{kg}$ BM, respectively. Participants were also provided with a post-exercise recovery drink, $20 \mathrm{~min}$ after the completion of the TT ( $1 \mathrm{~g} / \mathrm{kg} \mathrm{BM} \mathrm{CHO}$ and $25 \mathrm{~g}$ protein). The composition and timing of all meals was repeated prior to trial two.

\section{Synthesis of Ketone Ester}

The ketone ester synthesized, 1,3-butanediol acetoacetate diester, is a non-ionized sodium-free and $\mathrm{pH}$-neutral precursor of AcAc (D'Agostino et al., 2012). The ketone ester was synthesized by transesterification of $t$-butylacetoacetate with $R, S$-1,3-butanediol (Savind, Seymour, IL). The resultant product consisted of a mixture of monoesters and diester, the ratio of which could be adjusted by varying the stoichiometry of reactants. After synthesis the crude product was distilled under reduced pressure to remove all solvents and starting materials and the resultant ketone ester was obtained and assessed for purity using gas chromatography-mass spectrometry (GC-MS).

\section{Trial Day Procedure} Participant Preparation

On each of the trial days, participants reported to the laboratory in a rested and overnight $(10 \mathrm{~h})$ fasted state, with the timetable creating a $\sim 30$ min time between individuals that was repeated on the subsequent trial day. The trial day protocol commenced with the placement of an indwelling cannula (22G; Terumo, Tokyo, Japan) into a cephalic vein while lying in a supine position to allow for repeat blood sampling. A fingertip sample 
of capillary blood was collected concomitantly with each cannula sample throughout each trial for immediate measurement of blood ketones (ß-hydroxybutyrate; FreeStyleOptium Neo, Abbott Diabetes Care, Doncaster, Australia). Following a resting blood sample $(t=0 \mathrm{~min})$, participants were provided their standardized CHO breakfast including $200 \mathrm{mg}$ caffeine (NODOZ Awakeners, Key Pharmaceuticals Pty Ltd, Macquarie Park, Australia), to mimic typical race preparations. Participants were instructed to consume the breakfast within $10 \mathrm{~min}$, with a second blood sample being collected at $t=30 \mathrm{~min}$. At $t=70 \mathrm{~min}$, participants provided a urine sample, were weighed and fitted with a heart rate (HR) monitor. At this time, they ingested the first dose $(250 \mathrm{mg} / \mathrm{kg} \mathrm{BM})$ of KET or PLAC, followed immediately by $200 \mathrm{~mL}$ diet cola. At $t=90 \mathrm{~min}$ participants were seated on the Velotron ergometer, blood samples $(4 \mathrm{~mL})$ were collected and participants consumed their second dose in the same manner.

\section{Warm Up Protocol}

Following the second KET or PLAC drink, participants completed a standardized 20-min warm up on the cycle ergometer. The set-up of the bike was performed by team mechanics to replicate each rider's unique bicycle position and was fitted with a calibrated (Gardner et al., 2004) SRM cycling power meter (scientific version, 8 strain gauge, Schoberer Rad Meßtechnik; Jülich, Germany), set to sample at 1-s intervals. The warm up consisted of $3 \times 5 \mathrm{~min}$ at $50 \%$ ventilatory threshold (VT), VT1, and VT1 plus 50\% of the difference between VT1 and VT2 $(156 \pm 14,312 \pm 28,355 \pm 29 \mathrm{~W}$, respectively), followed by 5 min self-paced cycling. Venous and capillary blood samples were collected every $5 \mathrm{~min}$ and expired gas was collected continuously during the first $15 \mathrm{~min}$ of the warm up. Immediately following the warm up participants provided a urine sample and ingested an energy gel containing $50 \mathrm{mg}$ caffeine $(27 \mathrm{~g} \mathrm{CHO}$, PowerBarPowerGel). During this time ( $5 \mathrm{~min}$ ), participants were free to complete their own preparations during which pre-TT blood samples were collected, participants were provided with standard pre-race instructions and the zero offset of the SRM crank was set according to manufacturer's instructions.

\section{Cycling Time-Trial (World Championship Road Cycling Time Trial Simulation)}

The TT consisted of a simulation of the 2017 Bergen World Championship TT course, based on global positioning system (GPS) mapping data (road altitude and distance) collected by the Orica cycling team staff (M. Quod, unpublished observations). Cyclists completed the $31.17 \mathrm{~km}$ TT as fast as possible and during the TT the only feedback provided to the participant was the distance covered $(\mathrm{km})$, cycling gear-ratio (12-27/48-54) and road gradient (\%). Participants were only informed of their TT results following the completion of both trials. HR was collected every $5 \mathrm{~km}$ and ratings of perceived exertion (RPE) using the Borg 6-20 scale and capillary blood samples were collected at $15.74 \mathrm{~km}$ and immediately post TT. Participants ingested $250 \mathrm{~mL}$ of commercially available 6\% $\mathrm{CHO}$ drink (Gatorade) at 15.74 $\mathrm{km}$, as this distance corresponded to the point identified by the cyclists as the most appropriate opportunity to drink on the actual course. Samples of venous and capillary blood, and urine, were collected immediately following the TT and participants were weighed. At $t=\mathrm{TT}+20 \mathrm{~min}$, blood samples were collected and participants consumed a recovery drink ( $1 \mathrm{~g} / \mathrm{kg} \mathrm{BM} \mathrm{CHO})$ and continued to rest quietly for a further $40 \mathrm{~min}$. Blood samples were collected at $t=\mathrm{TT}+40 \mathrm{~min}$ and $t=\mathrm{TT}+60 \mathrm{~min}$, with a final urine sample being collected at $t=\mathrm{TT}+60 \mathrm{~min}$. Following the removal of the cannula, participants participated in a semi-structured interview with a single researcher using a series of standard questions to probe perceived effort, motivation and comfort rating during the TT. When symptoms (e.g., gut discomfort and problems) were identified, a standardized Likert scale was used to quantify them into mild, moderate, or severe levels. On completion of the second trial, participants were asked whether they could identify the trial in which they received the ketone ester, and the trial in which they had performed best. The interview technique was used to probe levels of interest in using a ketone ester supplement in real competition.

\section{Analytical Procedures}

Capillary blood samples were analyzed for concentration of ketones and lactate (Lactate Pro 2, Akray, Japan). Venous blood samples were collected into $4 \mathrm{~mL}$ SST vacutainers with immediate analysis of a small aliquot for blood glucose concentrations (Cobas Integra 400 plus, Roche Diagnostics, Switzerland). This venous sample was then centrifuged at $1,500 \mathrm{~g}$ for $10 \mathrm{~min}$ at $4^{\circ} \mathrm{C}$, and aliquots of serum were stored at $-80^{\circ} \mathrm{C}$ for later analysis. Samples were analyzed for FFA concentrations using a non-esterified-fatty acid (NEFA) assay kit (Wako Pure Chemical Industries, Ltd, Osaka, Japan), ßeta-hydroxybutyrate concentrations using a $\beta$-hydroxybutyrate assay kit (SigmaAldrich, Ltd, Australia) and acetoacetate (AcAc) concentrations using an acetoacetate assay kit (Abcam, Cambridge, UK), as per the manufacturer's instructions. Urine samples were analyzed for urine ketones (namely AcAc) using ketone reagent strips (Keto-Diastix, Bayer).

\section{Data Analysis}

Statistical analysis was completed using SPSS (version 20 for Windows; SPSS, Chicago, IL). Paired t-tests were used to analyze average $\mathrm{PO}$, cadence, HR and change in BM in the TT. Blood, urine, PO, HR, cadence, RPE, and respiratory data from the two trials were analyzed using a linear mixed model (treatment $\times$ time; $n=10$ ) with the exception of respiratory data which includes $(n=9)$. When analyzing respiratory gases, an RER $>1.0$ was not included in analysis as participants were not in steady state ( $n=1$, stage 3 for KET and PLAC). Statistical significance was set at $P<0.05$ and data is presented as mean \pm standard deviation $(S D)$. TT performance was also analyzed for magnitude-based effect sizes between conditions using a custom spreadsheet (Hopkins, 2006). Data were log-transformed to account for non-uniformity and effect sizes with $90 \%$ confidence intervals [effect size (ES) $\pm 90 \% \mathrm{CI}$ ] were calculated and classified as either trivial ( -0.2 to $0.2 \mathrm{ES})$ small $(0.2-0.6, \mathrm{ES})$, moderate (0.6-1.2 ES), or large (1.2-2.0 ES). Where the 90\% CI overlapped small positive (0.2) and negative (0.2) values, the effect was considered to be unclear. 


\section{RESULTS}

\section{Participant Experiences}

Eleven cyclists commenced this study, but one participant experienced severe side effects from KET ingestion during and after the warm-up, including prolonged vomiting and dizziness and was unable to complete the TT. This participant withdrew from further participation in the study. However, data for this participant have been provided in the following analysis in comparison to those of the other riders to investigate a possible explanation for the occurrence of these side effects. All participants reported gastrointestinal discomfort associated with the intake of the ketone diester. Symptoms ranged from major (dry retching and nausea; $n=2)$, to moderate nausea $(n=5)$ or moderate reflux $(n=1)$, and minor discomfort (mild nausea; $n=$ 2). No similar symptoms were reported with the PLAC trial. All participants correctly nominated the trial in which they received KET, identifying it via the gastrointestinal side-effects. However, only four of the cyclists correctly identified the trial in which they completed the TT in the fastest time, with one cyclist equivocal. Although each of the riders nominated their gut symptoms as a distraction or interference to performance, six participants identified an "unusual" centrally-derived feeling during the TT in the KET trial that they thought might be associated with better performance. When asked if they would use the current KET supplement in actual competition, prior to the unmasking of performance results, only one participant (who reported the least degree of discomfort during his KET trial) nominated being "possibly" interested. The remaining participants identified the need to remove the potential for illness and gut upset as well as to be sure of a robust performance effect before KET would be of value; "racing is hard enough without adding this complication."

\section{Performance}

All cyclists completed the TT in a faster time in PLAC compared with KET, with the crossover allocation of treatments meaning that there was no order effect on performance. Figure $\mathbf{2}$ displays the results of the cycling TT including group mean and individual performances. There was an impairment to overall performance time with KET $(2 \pm 1 \%, 58.2 \mathrm{~s}$; small ES $-0.42 \pm 0.1, P<0.001)$. There was an impairment in cycling performance time in the first segment of the course $(0-18 \mathrm{~km} ; P<0.001)$ and second segment with the climb included $(18-32 \mathrm{~km} ; P=0.004)$ with KET ingestion compared to PLAC. Overall the KET condition was associated with a $3.7 \%$ reduction in average PO (KET 339 $\pm 37 \mathrm{~W}$ vs. PLAC $352 \pm 35 \mathrm{~W}, P<0.001$, Figure 3B) and a lower cadence (KET $93 \pm 6 \mathrm{rpm}, \mathrm{PLAC} 95 \pm 6 \mathrm{rpm}, P=0.06$, Figure 3C) compared to PLAC. There was an effect of time $(P<$ 0.001 ) for power output and cadence during the TT, as displayed in Figure 3. A time $\times$ treatment interaction was reported for HR $(P=0.001)$ and average HR was significantly lower in the KET compared to PLAC condition ( $163 \pm 7$ vs. $167 \pm 9 \mathrm{bpm}$, respectively, $P<0.01$; Figure $3 \mathrm{D})$. RPE increased in both the KET $(16 \pm 2$ to $19 \pm 2)$ and the PLAC $(15 \pm 2$ to $19 \pm 1)$ trials from mid- to post-TT $(P<0.001)$. There was no difference in RPE between trials despite the lower HR and PO in the KET condition.

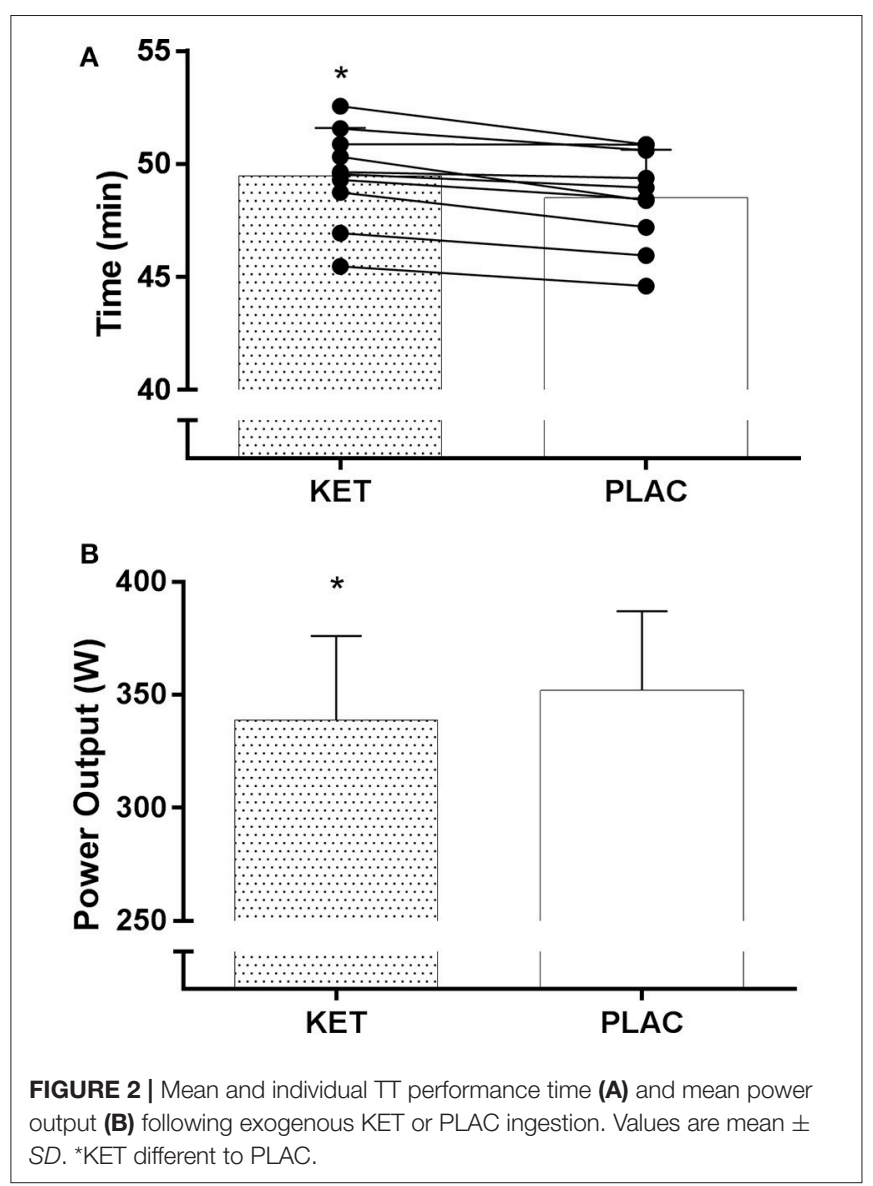

\section{Serum Metabolites}

There was an effect of time $(P<0.001)$ and treatment $(P=0.021)$ for serum FFA concentrations (Figure 4A). FFA concentrations reduced from $t=0$ during the $90 \mathrm{~min}$ following the $\mathrm{CHO}$ breakfast in both KET and PLAC trials $(0.37 \pm 0.10 \mathrm{mmol} / \mathrm{L}$ to $0.27 \pm 0.04 \mathrm{mmol} / \mathrm{L}, P<0.02)$. FFA concentrations were higher in PLAC vs. KET from pre- to post-TT $(P<0.05)$. A condition $\times$ time interaction was reported for serum $\beta \mathrm{HB}$ concentrations $(P<0.001$; Figure 4B). There was an increase in $\beta \mathrm{HB}$ concentrations in the KET trial following dose one of KET ingestion $(t=90 \mathrm{~min})$ and $\beta \mathrm{HB}$ remained significantly higher than PLAC trial until $t=\mathrm{TT}+60 \mathrm{~min}$. An increase in $\beta \mathrm{HB}$ concentrations from $t=0$ was measured in the PLAC trial at the onset of the warm up ( $t=100 \mathrm{~min}$; Figure $4 \mathrm{~B}$ ) however $\beta \mathrm{HB}$ remained lower than in the KET trial. Serum AcAc concentration significantly increased from $t=0 \mathrm{~min}$ following dose one of KET ingestion $(P=0.001)$ and remained higher until $t=\mathrm{TT}+60 \mathrm{~min}$ (Figure 4C).

\section{Capillary Blood and Urine Metabolites}

There was a condition $\times$ time interaction for both urine ketone and capillary blood $\beta \mathrm{HB}$ concentrations $(P<0.001$; Figures 5A,B). No differences were observed at $t=0$ between KET and PLAC for urine ketone concentration, but following KET ingestion, urine ketones were higher at pre-TT, post-TT, and at $t=\mathrm{TT}+60 \mathrm{~min}$ for KET. Blood $\beta \mathrm{HB}$ concentrations increased 

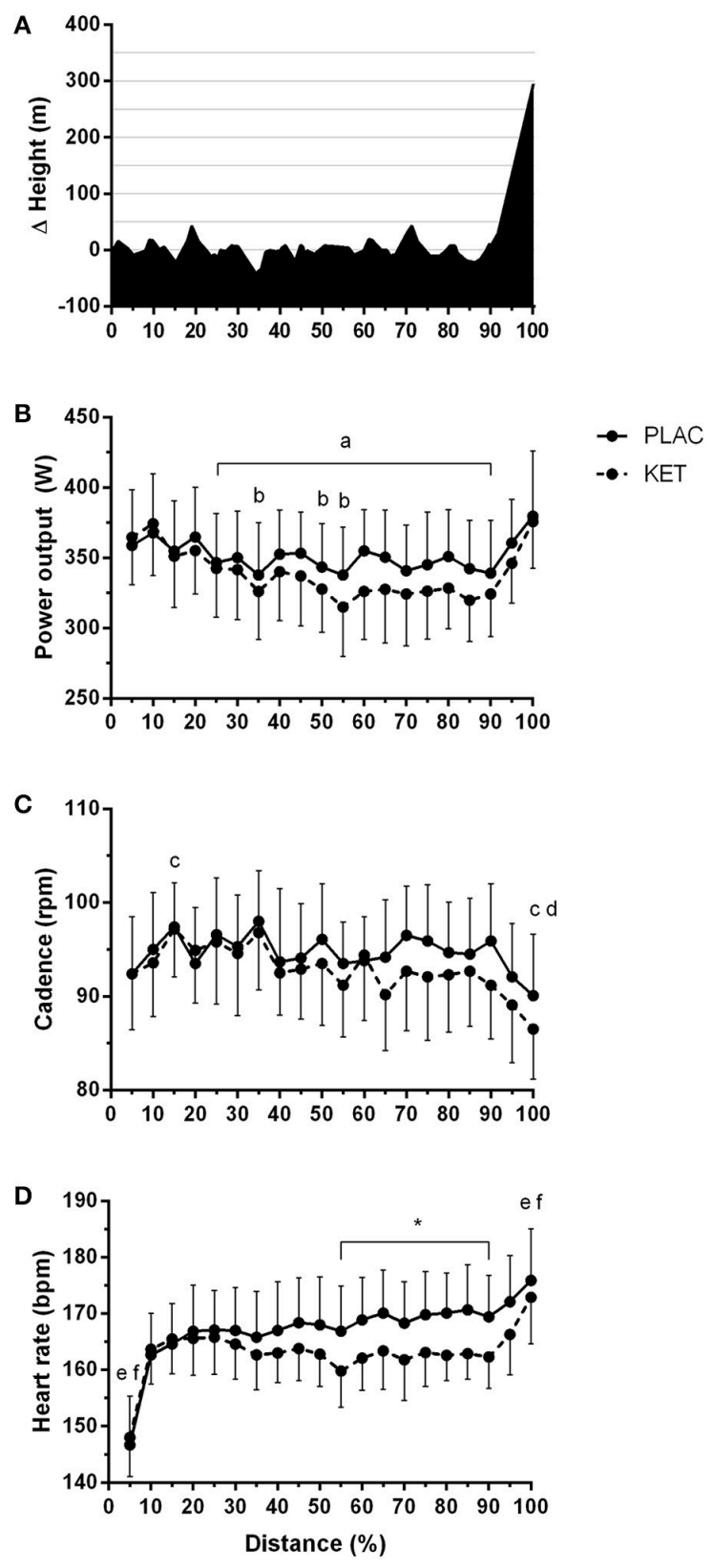

FIGURE 3 | Course profile of World Championships $\Pi$ course (Bergen, Norway) (A), average power output (B), cadence (C), and heart rate (D) during $\Pi$ as a percentage of total distance, following exogenous KET or PLAC ingestion. Values are mean $\pm S D$. a different to $5 \%$ in KET; b different to $5 \%$ in PLAC; c different to $10 \%$ in KET; d different to $10 \%$ in KET; ${ }^{*} \mathrm{KET}$ different to PLAC, e different to all other time points in KET, 50\% different to $f$.

following the first dose of KET, compared with PLAC ingestion $(0.32 \pm 0.16 \mathrm{mmol} / \mathrm{L}, P=0.001)$. Blood $\beta \mathrm{HB}$ concentrations increased from pre-TT to post-TT in the KET trial $(P<0.001)$ and this increase was maintained until $t=\mathrm{TT}+60 \mathrm{~min}(P=$ 0.03; Figure 5B).

There was a significant condition $\times$ time interaction for blood glucose $(P=0.036)$ and lactate concentrations $(P<0.001$; Figures 6A,B). Blood glucose concentrations were lower in KET
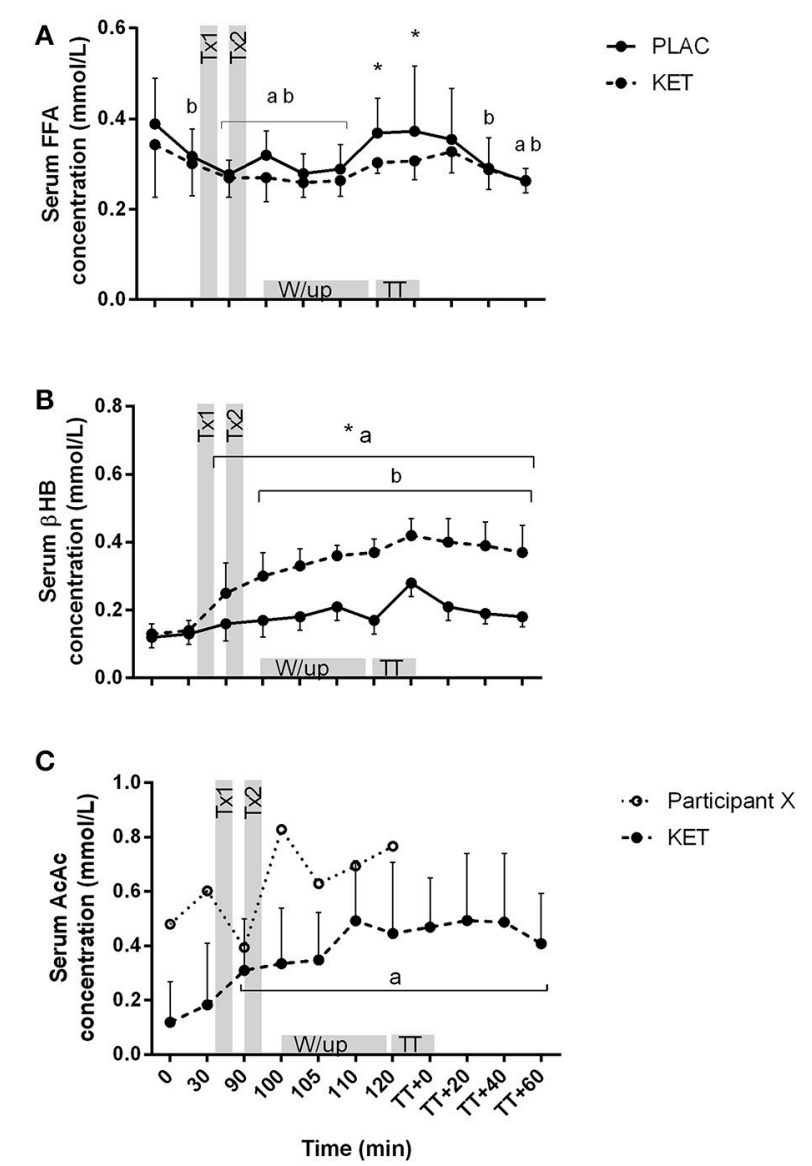

FIGURE 4 | Serum FFA (A), $\beta \mathrm{HB}$ (B), and AcAc (C) concentrations following exogenous KET or PLAC ingestion whilst completing a cycling $\Pi$. Values are mean $\pm S D$. Tx1 and Tx2 refer to dose one and two of KET or PLAC drink. *KET different to PLAC at time point; a different to $t=0$ min within KET; $\mathrm{b}$ different to $t=0$ min within PLAC.

following the first dose of KET ingestion, pre-TT and $t=\mathrm{TT}+$ 40 , compared to PLAC. Blood lactate concentrations increased from pre-warm up at the end of stage $3(t=110 \mathrm{~min})$ in both the KET and PLAC trials but had returned to resting values preTT for both trials. Post-TT, blood lactate concentrations were significantly lower in the KET trial compared to the PLAC trial $(8.6 \pm 3.2$ vs. $13.1 \pm 4.3 \mathrm{mmol} / \mathrm{L}, P<0.001$, respectively).

\section{Respiratory Parameters and BM}

There was a main effect of time in the KET and PLAC trials for $\mathrm{VO}_{2}, \mathrm{VCO}_{2}$, and $\operatorname{RER}(P<0.001)$, where an increase was observed throughout the incremental warm up from stage 1 to stage 3 (Table 1). There was a main effect of time for BM $(P$ $<0.001)$ where a similar loss was measured in the KET $(1.6 \pm$ $0.7 \mathrm{~kg})$ and PLAC $(1.4 \pm 0.4 \mathrm{~kg})$ trials.

\section{DISCUSSION}

This is the first study to report the effect of pre-exercise supplementation with a ketone diester on the performance of a cycling TT under conditions simulating real-life competition: 


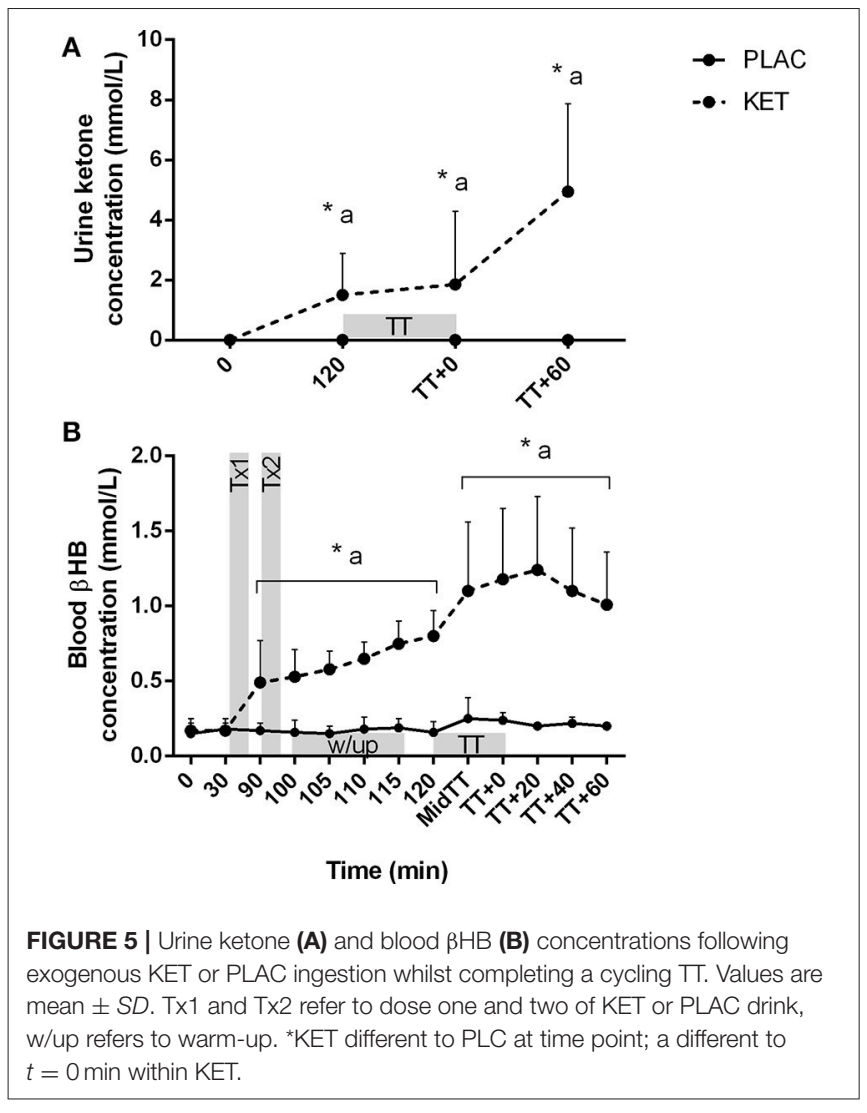

laboratory simulation of a World Championship TT course in world-class male road cyclists who undertook nutritional strategies mimicking competition practices with respect to $\mathrm{CHO}$ and caffeine supplementation. Although, our protocol achieved hyperketonemia, as evidenced by increases in serum $\beta \mathrm{HB}$ and AcAc concentrations, there was an impairment of TT cycling performance in these elite cyclists. This outcome appears to be linked to the general observation of gut discomfort and intolerance among the study participants, with symptoms ranging from mild to severe. Our investigation adds important information to sports nutrition, by adding a real-world element.

The primary aim of our study was to address recent reports of enhanced sports performance associated with an acute increase in blood ketone concentrations following the intake of a ketone ester drink (Cox et al., 2016), by re-examining this concept in a more ecologically valid protocol. The investigation was undertaken as a collaborative project with a World Tour professional team of the International Cycling Union (UCI), offering an opportunity for them to make an evidence-based decision regarding the potential use of a highly discussed performance aid (Abraham, 2015). A number of features were included in the study design to optimize the reliability and validity of data, including opportunities to mimic the conditions under which the performance aid (ketone ester supplement) would be used. Team sports scientists were able to provide a simulation of the profile of the 2017 World Championships TT course (Bergen, Norway) on a cycle ergometer. Furthermore,

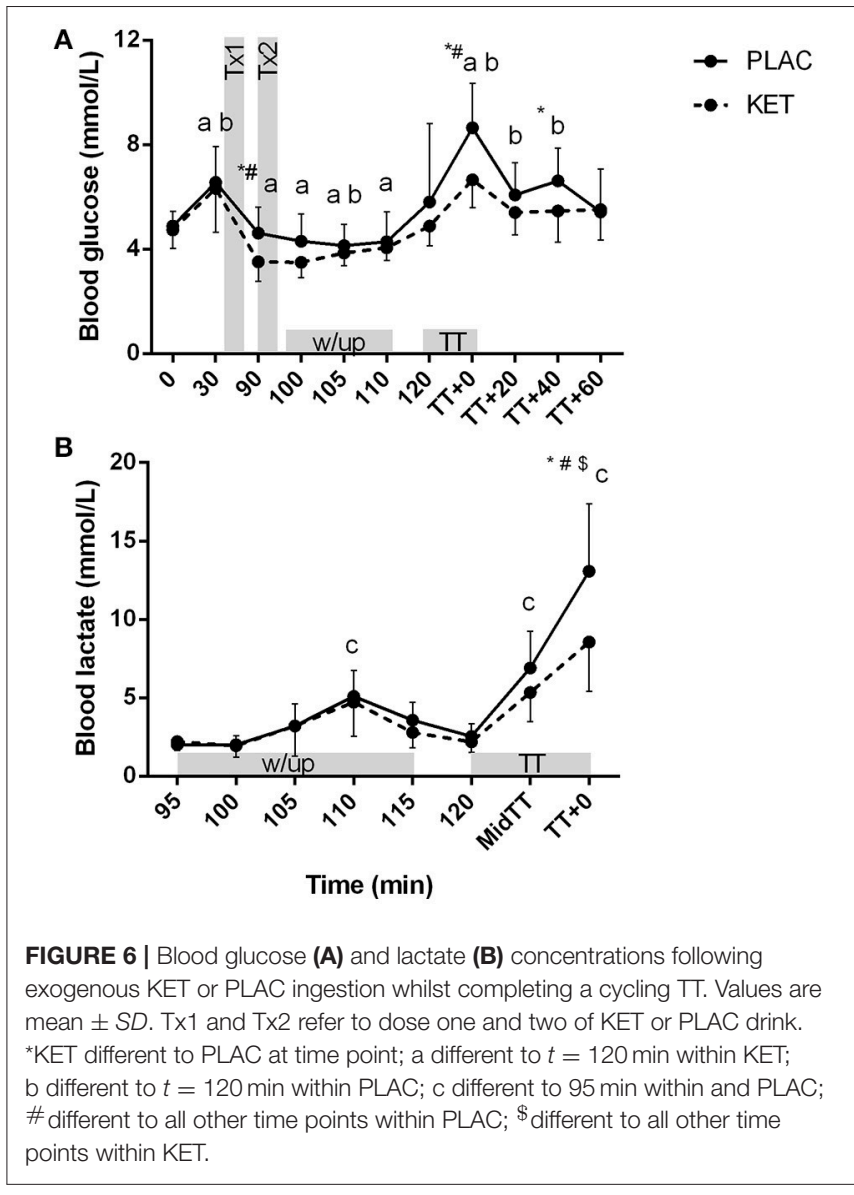

the world-class cyclists from the team who participated in the study were personally motivated to receive individual and group results, and were highly experienced in TT cycling as well as familiarized to the specific laboratory-based course simulation. Race nutrition strategies $(24 \mathrm{~h}$ prior, pre-race and during race) were standardized and made realistic by involving meal preparation by the team chef to suit both sports nutrition guidelines (Thomas et al., 2016) and the cultural practices of the riders. This included attention to achieve adequate $\mathrm{CHO}$ availability in pre-race meals (as opposed to the overnight fasted state involved in other studies such as Balasse, 1979; Fery and Balasse, 1986; Cox et al., 2016), as well as the real-life intake of caffeine and $\mathrm{CHO}$ supplements during the pre-race and withinrace practices.

Our primary finding of a $2.0 \pm 1 \%$ (58 s) longer time to completion in the TT following ketone ingestion supports our initial hypothesis that ketone ingestion would not enhance TT performance (Figure 2). Although, the gut disturbances were the likely cause of the performance impairment, we note that none of the cyclists achieved a faster time in the ketone trial, even when they reported very minor symptoms. Furthermore, the RPE in the ketone trial were similar to those on the placebo trial despite a lower power output and heart rate. This suggests that the gastrointestinal discomfort and/or some direct effect of ketones on the brain increased the perception of effort, and in accordance 
TABLE 1 | Metabolic measures collected during 3 stage incremental warm up following exogenous KET or PLAC ingestion.

\begin{tabular}{llccc}
\hline Variable & Treatment & Stage 1 & Stage 2 & Stage 3 \\
\hline $\mathrm{VO}_{2}(\mathrm{~L} / \mathrm{min})$ & KET & $2.27 \pm 0.27$ & $3.95 \pm 0.37^{\star}$ & $4.42 \pm 0.37$ \\
& PLAC & $2.33 \pm 0.26$ & $4.01 \pm 0.38^{\star}$ & $4.45 \pm 0.42$ \\
$\mathrm{VCO}_{2}(\mathrm{~L} / \mathrm{min})$ & KET & $1.94 \pm 0.21$ & $3.66 \pm 0.37^{\star}$ & $4.28 \pm 0.38$ \\
& PLAC & $2.02 \pm 0.24$ & $3.70 \pm 0.28^{\star}$ & $4.31 \pm 0.43$ \\
$\mathrm{RER}$ & KET & $0.86 \pm 0.04$ & $0.93 \pm 0.03^{\star}$ & $0.96 \pm 0.02$ \\
& PLAC & $0.87 \pm 0.03$ & $0.93 \pm 0.04^{\star}$ & $0.96 \pm 0.03$
\end{tabular}

${ }^{\star}$ Different to stage 1 and stage 3; Values are mean $\pm S D$.

with the psychobiological model of pacing (Pageaux et al., 2014), our highly experienced cyclists reduced their work output to enable the TT to be completed without premature exhaustion. This finding warrants further investigation to confirm and explore the mechanisms. In the meantime, we note that the outcome of impaired performance with ketone ingestion is in contrast to previous studies (Clarke and Cox, 2015; Cox et al., 2016). Indeed, Clarke and Cox (2015) and Cox et al. (2016) reported 1-2\% improvements in 30 min rowing performance and $30 \mathrm{~min}$ TT performance, respectively, following ingestion of a similar ketone dose to the current study (573 vs. $500 \mathrm{mg} / \mathrm{kg}$ $\mathrm{BM}$, respectively) in combination with $\mathrm{CHO}$, compared to $\mathrm{CHO}$ alone.

Due to the lack of a commercial supply, we were unable to obtain the ketone monoester supplement used in the study of Cox et al. (2016). However, we were able to source a diester that is currently being investigated as a potential treatment for seizures resulting from central nervous system oxygen toxicity (D'Agostino et al., 2013) and used in a similar dose to Cox et al. (2016). This diester contains a racemic mixture of $\beta \mathrm{HB}$ (i.e., contains both $\mathrm{D}$ - and L- enantiomers of the $\beta \mathrm{HB}$ ) and has the ability to elevate both $\beta \mathrm{HB}$ and AcAc in a 1:1 ratio. The use of enzymatic analysis in the current study measures only the $\mathrm{D}$ enantiomer which is the main circulating form of $\beta \mathrm{HB}$ and the most likely to have a direct effect on substrate metabolism and skeletal muscle responses (Yamada et al., 2010).

We provided the ketone drink in two doses, with the first bolus ingested $70 \mathrm{~min}$ prior to the TT. Based on previous research, our aim was to reach peak $\beta \mathrm{HB}$ concentrations at $\sim 1 \mathrm{~h}$ following ingestion (i.e., prior to the TT; Pinckaers et al., 2017). We measured a modest increase in serum $\beta \mathrm{HB}$ concentrations in the ketone trial, reaching $>0.3 \mathrm{mmol} / \mathrm{L}$ following the warm-up, but to our surprise serum $\beta \mathrm{HB}$ concentrations peaked immediately following the TT $(>0.4 \mathrm{mmol} / \mathrm{L}$; Figure $4 \mathrm{~B})$. The capillary whole blood samples analyzed for $\beta \mathrm{HB}$ concentrations measured values 2 - to 3 -fold greater than the serum samples (Figure 5B). This variation in $\mathrm{D}-\beta \mathrm{HB}$ concentrations via enzymatic analysis (serum) and whole blood is consistent with previous literature reporting a -0.5 to $0.6 \mathrm{mmol} / \mathrm{L}$ higher concentration with handheld monitors (Pineda and Cardoso, 2015). This variation in measuring blood ketones in a controlled laboratory setting highlights the challenges athletes face in the field when aiming to reach and stay within the "optimal" range for a performance benefit (Egan and D'Agostino, 2016). We also measured a peak in serum AcAc concentrations following the warm-up, reaching $\sim 0.5 \mathrm{mmol} / \mathrm{L}$ (Figure 4C). Therefore, when we consider total circulating ketones measured (i.e., $\beta \mathrm{HB}$ and AcAc) and the L- enantiomer that has not been measured, it is likely that athletes would be in the "optimal" range of 1-3 mmol/L for a proposed performance benefit (Egan and D'Agostino, 2016). Although "nutritional ketosis" was achieved, $\beta \mathrm{HB}$ concentrations reported in the current study are much lower than those reported previously (Cox et al., 2016). Cox et al. (2016) reported an increased in $\beta \mathrm{HB}$ concentrations to $\sim 2 \mathrm{mmol} / \mathrm{L}$ within 20 min of ketone ester ingestion when co-ingested with $\mathrm{CHO}$ or $\sim 4 \mathrm{mmol} / \mathrm{L}$ when ingested alone. This variation in serum $\beta \mathrm{HB}$ is likely explained by a range of factors including the different ketone esters used, the elite training status of cyclists in the current study and the different pre-ingestion nutritional strategies were the current study focused on appropriate race preparation practices.

Although we have not measured the same increase in circulating ketone concentrations as (Cox et al., 2016), ketones increased appropriately to alter metabolic responses compared to when a placebo was ingested. Blood glucose concentration was lowered in the ketone trial by $\sim 1 \mathrm{mmol} / \mathrm{L}$ within $30 \mathrm{~min}$ following ingestion of the first ketone dose, and following the TT blood glucose was $\sim 2 \mathrm{mmol} / \mathrm{L}$ lower than in the placebo trial. Additionally, we reported a $4.5 \mathrm{mmol} / \mathrm{L}(35 \%)$ reduction in blood lactate concentration following the TT in the ketone trial compared to the placebo trial. These findings of reduced blood glucose and blood lactate concentrations are consistent with the data of Cox et al. (2016) during 60 min of exercise at $75 \% \mathrm{Wmax}$ and following a $30 \mathrm{~min} \mathrm{TT}$, respectively. We also measured lower circulating FFA during the TT following ketone ingestion compared to a placebo. Participants were cycling at 340-350 W during the TT and thus estimated contribution of FFA oxidation to total energy expenditure would likely be low as at this intensity, the muscle relies predominately on $\mathrm{CHO}$ based fuels (Hawley and Leckey, 2015). However, the difference in FFA concentration between the ketone and placebo trials could be related to circulating ketone bodies having the ability to suppress lipolysis via inhibition of catecholamine's (Bjorntorp and Schersten, 1967).

As ketone bodies can be readily oxidized by skeletal muscle, expired gas was collected during the incremental warm-up. No differences in RER were measured between the ketone and placebo trials, although this could be related to the absolute exercise intensities attained by our elite subjects (155, 310, $355 \mathrm{~W})$. Alternatively the high ketone concentrations in the urine suggest that the ketones are not being oxidized at the skeletal muscle. We have not estimated rates of substrate oxidation rates due to $\beta \mathrm{HB}$ and AcAc yielding respiratory exchange quotient values of 0.89 and 1.00 , respectively and thus without appropriate correction factors for $\mathrm{CO}_{2}$ displacement and urine volume this would lead to an inaccurate representation of substrate utilization (Frayn, 1983; Pinckaers et al., 2017). We also reported a modest reduction in heart rate during the $\mathrm{TT}$ in the ketone trial compared to the placebo trial $(5 \mathrm{bpm})$ which may be associated with a slightly reduced average power output and in the ketone trial. 
Of the 10 participants who completed the trials, all reported gastrointestinal discomfort associated with the intake of the ketone ester including dry retching, mild to moderate nausea, reflux and minor discomfort. Furthermore, one participant was unable to start the TT due to prolonged vomiting and dizziness. This participant also experienced the highest concentrations of serum AcAc concentration when compared to the other 10 participants (Figure 4C, participant X), suggesting bioavailability of the ketone diester may impact individual responses following ketone ingestion. Although the side effects of ketone esters are not frequently discussed in the literature, Clarke et al. (2012) has provided evidence that participants have experienced a range of adverse effects including vomiting, nausea, diarrhea, and abdominal pain. These side effects have been associated with high dose of ketone ester and the consumption of the ester with a milk-based drink (Clarke and Cox, 2015). While it is possible that different dosing strategies, or the use of a different ketone ester product might eliminate or greatly reduce the gut problems seen in the current study, it is unclear whether a performance enhancement could be expected with exogenous ketone use in sporting events undertaken under the conditions employed in our study.

In conclusion, the results of the current study show that ingestion of a 1,3-butanediol acetoacetate diester under conditions of optimal race nutrition (i.e., $\mathrm{CHO}$ fed) results in increases in $\beta \mathrm{HB}$ and $\mathrm{AcAc}$ concentrations. The diester was associated with gut discomfort and intolerance among the cyclists with symptoms ranging from mild to severe. Despite optimal nutritional support (i.e., $\mathrm{CHO}$ breakfast, feeding during the TT and caffeine ingestion) for performance, ketone ingestion was associated with an increase in perception of effort, leading

\section{REFERENCES}

Abraham, R. (2015). Ketones: Controversial New Energy Drink Could Be Next Big Thing in Cycling. Available online at: http://www.cyclingweekly.co.uk/news/ latest-news/ketones-controversial-new-energy-drink-nextbig-thing-cycling151877 (Accessed July 24, 2015).

Balasse, E. O. (1979). Kinetics of ketone body metabolism in fasting humans. Metab. Clin. Exp. 28, 41-50. doi: 10.1016/0026-0495(79)90166-5

Balasse, E., and Ooms, H. A. (1968). Changes in the concentrations of glucose, free fatty acids, insulin and ketone bodies in the blood during sodium beta-hydroxybutyrate infusions in man. Diabetologia 4, 133-135. doi: 10.1007/BF01219433

Bjorntorp, P., and Schersten, T. (1967). Effect of beta-hydroxybutyrate on lipid mobilization. Am. J. Physiol. 212, 683-687.

Brooks, G. A., and Mercier, J. (1994). Balance of carbohydrate and lipid utilization during exercise: the crossover concept. J. Appl. Physiol. 76, 2253-2261.

Burke, L. M., Ross, M. L., Garvican-Lewis, L. A., Welvaert, M., Heikura, I. A., Forbes, S. G., et al. (2017). Low carbohydrate, high fat diet impairs exercise economy and negates the performance benefit from intensified training in elite race walkers. J. Physiol. 595, 2785-2807. doi: 10.1113/ JP273230

Clarke, K., and Cox, P. (2015). Ketone Bodies and Ketone Body Esters for Maintaining or Improving Muscle Power Output (Application no WO20150164855 A1). Oxfordshire, UK: European Patent Office.

Clarke, K., Tchabanenko, K., Pawlosky, R., Carter, E., Todd King, M., Musa-Veloso, K., et al. (2012). Kinetics, safety and tolerability of (R)-3-hydroxybutyl (R)-3-hydroxybutyrate in healthy adult subjects. Regul. Toxicol. Pharmacol. 63, 401-408. doi: 10.1016/j.yrtph.2012. 04.008 to an impairment of TT performance in elite professional cyclists.

\section{AUTHOR CONTRIBUTIONS}

JL, MR, MQ, and JH: Conception and design, Collection and assembly of data, Data analysis and interpretation, Manuscript writing, Final approval of manuscript (required). LB: Conception and design, Financial support, Collection and assembly of data, Data analysis and interpretation, Manuscript writing, Final approval of manuscript (required).

\section{FUNDING}

This study was funded by a Program Grant from the Australian Catholic University Research Funds to LB (ACURF. 2017000034).

\section{ACKNOWLEDGMENTS}

The authors would like to thank volunteers from the Australian Institute of Sport (Julia Bone, Tony Rice, Kate Fuller, Hamilton Lee, Alice Wallett, Jess Sewastenko, Avish Sharma, Margot Rogers, Rebekah Alcock, Chris Fonda, Melissa Holloway, Bronwen Lundy, Nicolin Tee, Victor Vuong, and Jamie Plowman), ORICA-BikeExchange (Paolo Menaspa, Nicki Strobel, Wayne Nichols, Craig Geater) and Cycling Australia (Olivia Warnes), for their assistance during data collection. The collaborative involvement of the ORICA-BikeExchange cyclists is gratefully acknowledged. We also thank Professor Dominic D’Agostino for making the ketone diester available for this work.

Cox, P. J., Kirk, T., Ashmore, T., Willerton, K., Evans, R., Smith, A., et al. (2016). Nutritional ketosis alters fuel preference and thereby endurance performance in athletes. Cell Metab. 24, 256-268. doi: 10.1016/j.cmet.2016. 07.010

D'Agostino, D. P., Dean, J. B., and Pilla, P. A. R. (2012). Use of Ketone Esters for Prevention of CNS Oxygen Toxicity (Application no WO 2012154837 A1). Florida: US Patent Office.

D’Agostino, D. P., Pilla, R., Held, H. E., Landon, C. S., Puchowicz, M., Brunengraber, H., et al. (2013). Therapeutic ketosis with ketone ester delays central nervous system oxygen toxicity seizures in rats. Am. J. Physiol. Regul. Integr. Comp. Physiol. 304, R829-R836. doi: 10.1152/ajpregu. 00506.2012

Egan, B., and D'Agostino, D. P. (2016). Fueling performance: ketones enter the mix. Cell Metab. 24, 373-375. doi: 10.1016/j.cmet.2016.08.021

Evans, M., Cogan, K. E., and Egan, B. (2017). Metabolism of ketone bodies during exercise and training: physiological basis for exogenous supplementation. J. Physiol. 595, 2857-2871. doi: 10.1113/JP273185

Fery, F., and Balasse, E. O. (1986). Response of ketone body metabolism to exercise during transition from postabsorptive to fasted state. Am. J. Physiol. 250(5 Pt 1), E495-E501.

Frayn, K. N. (1983). Calculation of substrate oxidation rates in vivo from gaseous exchange. J. Appl. Physiol. Respir. Environ. Exerc. Physiol. 55, 628-634.

Gardner, A. S., Stephens, S., Martin, D. T., Lawton, E., Lee, H., and Jenkins, D. (2004). Accuracy of SRM and power tap power monitoring systems for bicycling. Med. Sci. Sports Exerc. 36, 1252-1258. doi: 10.1249/01.MSS.0000132380.21785.03

Hawley, J. A., and Leckey, J. J. (2015). Carbohydrate dependence during prolonged, intense endurance exercise. Sports Med. 45(Suppl. 1), S5-S12. doi: 10.1007/s40279-015-0400-1 
Hawley, J. A., Maughan, R. J., and Hargreaves, M. (2015). Exercise metabolism: historical perspective. Cell Metab. 22, 12-17. doi: 10.1016/j.cmet.2015.06.016

Hopkins, W. (2006). Spreadsheets for analysis of controlled trials with adjustment for a predictor. Sport Sci. 10, 46-50. Available online at: sportsci.org/2006/ wghcontrial.htm

Pageaux, B., Lepers, R., Dietz, K. C., and Marcora, S. M. (2014). Response inhibition impairs subsequent self-paced endurance performance. Eur. J. Appl. Physiol. 114, 1095-1105. doi: 10.1007/s00421-014-2838-5

Pinckaers, P. J., Churchward-Venne, T. A., Bailey, D., and van Loon, L. J. (2017). Ketone bodies and exercise performance: the next magic bullet or merely hype? Sports Med. 47, 383-391. doi: 10.1007/s40279-016-0577-y

Pineda, A., and Cardoso, F. C. (2015). Technical note: validation of a handheld meter for measuring beta-hydroxybutyrate concentrations in plasma and serum from dairy cows. J. Dairy Sci. 98, 8818-8824. doi: 10.3168/jds. 2015-9667

Robinson, A. M., and Williamson, D. H. (1980). Physiological roles of ketone bodies as substrates and signals in mammalian tissues. Physiol. Rev. 60, 143-187.

Russell, G., Gore, C. J., Ashenden, M. J., Parisotto, R., and Hahn, A. G. (2002). Effects of prolonged low doses of recombinant human erythropoietin during submaximal and maximal exercise. Eur. J. Appl. Physiol. 86, 442-449. doi: 10.1007/s00421-001-0560-6
Thomas, D. T., Erdman, K. A., and Burke, L. M. (2016). American college of sports medicine joint position statement. Nutrition and athletic performance. Med. Sci. Sports Exerc. 48, 543-568. doi: 10.1249/MSS.0000000000000852

Veech, R. L. (2004). The therapeutic implications of ketone bodies: the effects of ketone bodies in pathological conditions: ketosis, ketogenic diet, redox states, insulin resistance, and mitochondrial metabolism. Prostaglandins Leukot. Essent. Fatty Acids 70, 309-319. doi: 10.1016/j.plefa.2003.09.007

Yamada, T., Zhang, S. J., Westerblad, H., and Katz, A. (2010). $\beta$-Hydroxybutyrate inhibits insulin-mediated glucose transport in mouse oxidative muscle. Am. J. Physiol. Endocrinol. Metab. 299, E364-E373. doi: 10.1152/ajpendo.00142.2010

Conflict of Interest Statement: The authors declare that the research was conducted in the absence of any commercial or financial relationships that could be construed as a potential conflict of interest.

Copyright (๑ 2017 Leckey, Ross, Quod, Hawley and Burke. This is an open-access article distributed under the terms of the Creative Commons Attribution License (CC $B Y)$. The use, distribution or reproduction in other forums is permitted, provided the original author(s) or licensor are credited and that the original publication in this journal is cited, in accordance with accepted academic practice. No use, distribution or reproduction is permitted which does not comply with these terms. 